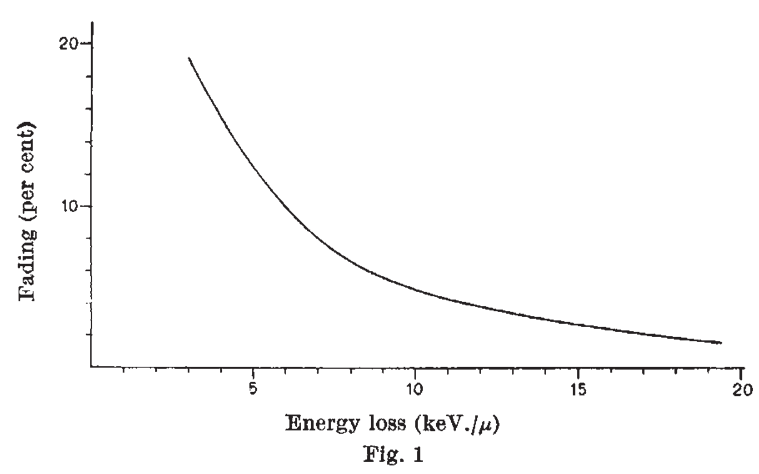

culated from the measurements of the earlier communication ${ }^{1}$. This explains the apparent increase in the mass-ratio (see Fig. 1 of ref. 1). The fact that the curve ceases to rise beyond a certain range depends on the method of calculation in such a way that the increase in range more than compensates for the diminution of grain density through fading.

This explanation is based on: (1) the published measurements ; (2) a series of plates with approximately the same history but which were more strongly developed ; the apparent mass-ratio in these plates was 1.6 ; (3) a series of exposed plates which were stored so that fading could not occur; the massratio obtained in this case was the correct one; (4) investigations by G. Leide (private communication) of fading in 65 emulsions.

The excessive density of proton tracks relative to the $\pi$-meson density appears to be caused by the fact that the resolution into protons, deuterons and tritons was uncertain in the first photoelectric measurements, and the value given for the proton density was a composite one obtained from both proton and deuteron tracks.

Department of Physics,

Krister Kristiansson

University of Lund. Sept. 22.

${ }^{1}$ von Friesen and Kristiansson, Nature, 168, 377 (1951). ${ }^{2}$ Gustafsson, Nature, 168, 377 (1951).

${ }^{8}$ Albouy and Faraggi, J. Phys. et le Rad., 10, 105 (1.949).

\section{Occurrence of a Fission-Dust Particle in a Nuclear Emulsion}

IN the microscopic examination of an Ilford Nuclear Research emulsion exposed to cosmic radiation in the stratosphere, an unusual radioactive inclusion was noted. As shown in the photomicrograph (Fig. 1), the embedded particle is strongly beta-active and when examined under conditions of higher resolution the tracks originate from an opaque, nearly spherical granule with a diameter of 5-10 microns. One alpha-particle track with a recorded range of $14 \pm 1$ microns is associated with the embedded grain. The fused appearance of the inclusion, together with its beta- and alpha-activity, suggests that it may have originated from an atomic weapons detonation.

The grain is embedded 125 microns below the air interface of a 1,500 -micron $Q 5$ plate manufactured at Ilford, London, on January 16, 1952, which was developed on March 30, 1952. Based on a diameter of 10 microns, an exposure period of 74 days, an assumed density of $3 \mathrm{gm}$. $\mathrm{cm}^{-3}$ and a count of

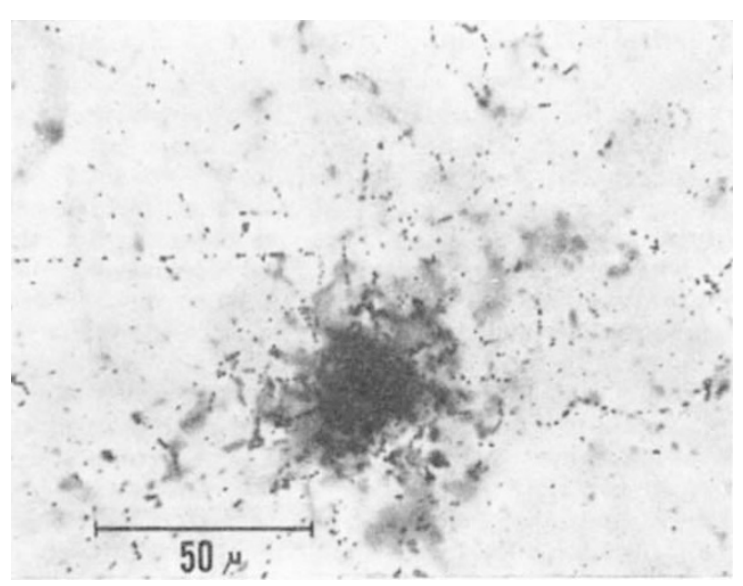

Fig. 1. Photomicrograph of flssion-colloid embedded in an

$100 \pm 50$ recorded electron tracks, the average specific activity of the inclusion over the period between manufacture and development is estimated at $10^{4}$ beta-decays per sec. per gm. This is a lower limit based on an apparent diameter the exact extremities of which are poorly defined owing to the large number of developed silver grains near the surface of the spherule. The true diameter might be as small as 5 microns, which would increase the estimate of the activity eightfold.

The position of this fission-colloid suggests that it was incorporated in the emulsion during manufacture as a contamination in either the gelatin, silver compounds, or the water. The active grain may conceivably have been airborne, settling on the surface of the freshly poured plate and sinking to a depth of 125 microns, where it remained suspended when the warm emulsion cooled to its jelling point.

This fission-type occlusion is the only one noted in the examination of 29 c.c. of emulsion of the same batch (Z5699). In all, about 300 c.c. of electronsensitive emulsion has been scanned in this laboratory, in which twenty-four polonium radio-colloid aggregates and six alpha-radioactive macro-inclusions have been noted. The latter are chiefly translucent grains 6-33 microns in diameter associated with an aura of alpha-particle tracks the ranges of which suggest an origin from either the radium or thorium series. Their specific activity varies between 170 and 13,500 alpha-decays per sec. per gm., which is roughly $10^{4}$ times greater than the alpha-activity of the rocks of the earth's crust. The activity of these macro inclusions appears to diminish with increase in particle size, suggesting that the activity originates from a surface adsorption deposit.

Collectively, the naturally occurring alpha-active inclusions occur with a frequency of 0.1 per c.c. of emulsion. If we assume that these bodies were introduced into the emulsion chiefly via the gelatin component, their concentration is increased to 0.2 per c.c., and this value may be indicative of the approximate abundance of strongly alpha-active segregates in normal biological tissues. A more detailed account of this study will be published elsewhere.

National Institutes of Health,

HERMAN YAGODA

$$
\text { Oct. } 9 .
$$

